\title{
What Factors Contribute to Worse Quality of Life in Thyroid Cancer Survivors?
}

\section{Tracy S. Wang}

Section of Endocrine Surgery, Department of Surgery, Medical College of Wisconsin, Milwaukee, Wisconsin, U.S.A.

Review of: Goswami S, Peipert BJ, Mongelli MN, Kurumety SK, Helenowski IB, Yount SE, Sturgeon C 2019. Clinical factors associated with worse quality-of-life scores in United States thyroid cancer survivors 2019 Surgery. Epub 2019 Mar 18. pii: S0039-6060(19)30066-2. PMID: 30898373.

\section{SUMMARY}

\section{Background}

The population of thyroid cancer survivors is increasing rapidly, given the increase in the diagnosis of thyroid cancer and the excellent survival rates for most patients. However, recent studies have suggested that self-reported health-related quality of life (HRQOL) is similar, if not worse, than in patients that are diagnosed with cancers with poorer prognoses. The study hypothesis was that more aggressive treatments and related adverse events would be associated with poorer HRQOL scores in thyroid cancer patients; the Patient-Reported Outcomes Measurement Information System (PROMIS) was used for this analysis (1).

\section{Methods}

PROMIS is a 29-item profile measure that evaluates mental, physical, and social health across seven domains. Higher scores for the domains of anxiety, depression, fatigue, pain interference, and sleep disturbance indicate worse HRQOL, while lower scores in the domains of physical function and ability to participate in social roles (social function) indicate worse HRQOL. Scores for each domain were generated using the Health Measures Scoring Service, and multivariable linear regression models were created to evaluate the impact of clinical and demographic factors on PROMIS T-scores. A P value of $<0.01$ was used to identify statistical significance, and a difference of 3 or more points was considered a clinically meaningful difference.

The survey was distributed in collaboration with the Thyroid Cancer Survivors' Association (ThyCAT) from January to June 2017 via several online methods. Eligible patients included those with a reported diagnosis of thyroid cancer, who resided in the United States, and were 18 to 89 years of age. After electronic consent was obtained, the participants were given the two-part survey online via Research Electronic Data Capture (REDCap).

\section{Results}

The survey was accessed by 3174 individuals, and both portions were completed by 1922 individuals (61\% participation rate); the final cohort consisted of 1743 eligible individuals. The majority of patients had papillary thyroid cancer (85\%), with most reported having stage 1 disease (30\%); $98 \%$ of participants underwent surgery and $78 \%$ underwent treatment with radioactive iodine (RAI).

The most common adverse effects of surgery were dysphagia (63\%) and dysphonia (71\%) lasting days to weeks after surgery; $47 \%$ reported transient and permanent hypocalcemia. The most common shortterm side effects of RAI reported were mild xerostomia (34\%) and taste or appetite changes (29\%), while 

Quality of Life in Thyroid Cancer Survivors?

the long-term side effects reported were chronic xerophthalmia (24\%) and xerostomia (24\%).

Demographic and clinical factors (including disease and treatment factors) were associated with worse HRQOL scores across multiple PROMIS domains. Patient age $<45$ years was associated with worse scores in domains of anxiety, depression, and fatigue, while dysphonia and dysphagia were associated with worse scores for the domain of fatigue. Surgical site infection, dysphagia, and short-term xerophthalmia were associated with higher scores for pain; surgical-site infection and long-term dental caries after RAI were associated with worse scores for social functioning. Impaired physical functioning scores were associated with treatment with chemotherapy, surgical-site infection, and the development of pulmonary fibrosis after RAI. No factors were significant for the domain of sleep disturbance.

\section{Conclusions}

Factors associated with worse HRQOL scores across multiple PROMIS domains in thyroid cancer survivors included age $<45$ years, postoperative hypocalcemia, dysphonia, dysphagia, appearance of the scar, and complications (both short- and longterm) from radioactive iodine.

\section{COMMENTARY}

Although the incidence of thyroid cancer is increasing, death from thyroid cancer has remained stable over the past several decades, owing to the excellent prognosis for the majority of patients with differentiated thyroid cancer (2). Perhaps as a result of this, until more recently, issues related to long-term quality of life (QOL) for survivors have not fully been evaluated (3-6). Recent studies have focused on evaluation of QOL in patients with thyroid cancer, with a focus on issues specific to thyroid disease and thyroid surgery, although there is not yet consensus as to an optimal or singular validated method (1,3-6).

This is one of the first studies to describe clinical factors associated with decreased HRQOL scores in thyroid cancer patients using the PROMIS instrument. The advantages of PROMIS include its validation across the general population as well as a variety of populations with cancer and chronic disease, which potentially allows for accurate comparison among different disease processes. For example, the results of this study are in agreement with previous studies using other instruments, such as ThyCAT, including the association between younger patients and worse HRQOL scores for depression, anxiety, and fatigue.

Disadvantages of the use of PROMIS is the inability to assess HRQOL specifically related to thyroid disease and, in particular, the consequences of thyroidectomy such as levothyroxine replacement and complications such as recurrent laryngeal-nerve injury and/or hypoparathyroidism, either transient or permanent. Thyroid-specific questionnaires have been developed, including ThyCAT, a QOL computerized adaptive test, and a core questionnaire being developed by the European Organisation for Research and Treatment of Cancer (EORTC) $(3,6)$.

It is likely that a combination of HRQOL instruments such as PROMIS, as well as thyroid diseasespecific instruments, will provide the most nuanced data regarding $\mathrm{HRQOL}$ in patients with thyroid cancer. These data are important, as physicians' perspectives on QOL issues may not parallel those of patients, and knowing patients' perspective on their posttreatment QOL will be a critical component of shaping management guidelines for the future (5). 

Quality of Life in Thyroid Cancer Survivors?

\section{REFERENCES}

1. Goswami S, Peipert BJ, Mongelli MN, Kurumety SK, Helenowski IB, Yount SE, Sturgeon C 2019 Clinical factors associated with worse quality-of-life scores in United States thyroid cancer survivors. Surgery. Epub 2019 Mar 18. pii: S0039-6060(19)30066-2.

2. Lubitz CC, Sosa JA 2016 The changing landscape of papillary thyroid cancer: epidemiology, management, and the implications for patients. Cancer 122:3754-3759. Epub 2016 Aug 12.

3. Aschebrook-Kilfoy B, Ferguson BA, Angelos P, Kaplan EL, Grogan RH, Gibbons RD 2018 Development of the ThyCAT: A clinically useful computerized adaptive test to assess quality of life in thyroid cancer survivors. Surgery 163:137-142. Epub 2017 Nov 8.
4. Goldfarb M, Casillas J 2016 Thyroid cancer-specific quality of life and health-related quality of life in young adult thyroid cancer survivors. Thyroid 26:923-932. Epub 2016 Jun 6.

5. James BC, Aschebrook-Kilfoy B, White MG, Applewhite MK, Kaplan SP, Angelos P, Kaplan EL, Grogan RH 2018 Quality of life in thyroid cancerassessment of physician perceptions. J Surg Res 226:94-99. Epub 2018 Feb 15.

6. Singer S, Jordan S, Locati LD, Pinto M, Tomaszewska IM, Araujo C, Hammerlid E, Vidhubala E, Husson O, Kiyota N, et al. 2017 The EORTC module for quality of life in patients with thyroid cancer: phase III. Endocr Relat Cancer 24:197-207. Epub 2017 Feb 21.

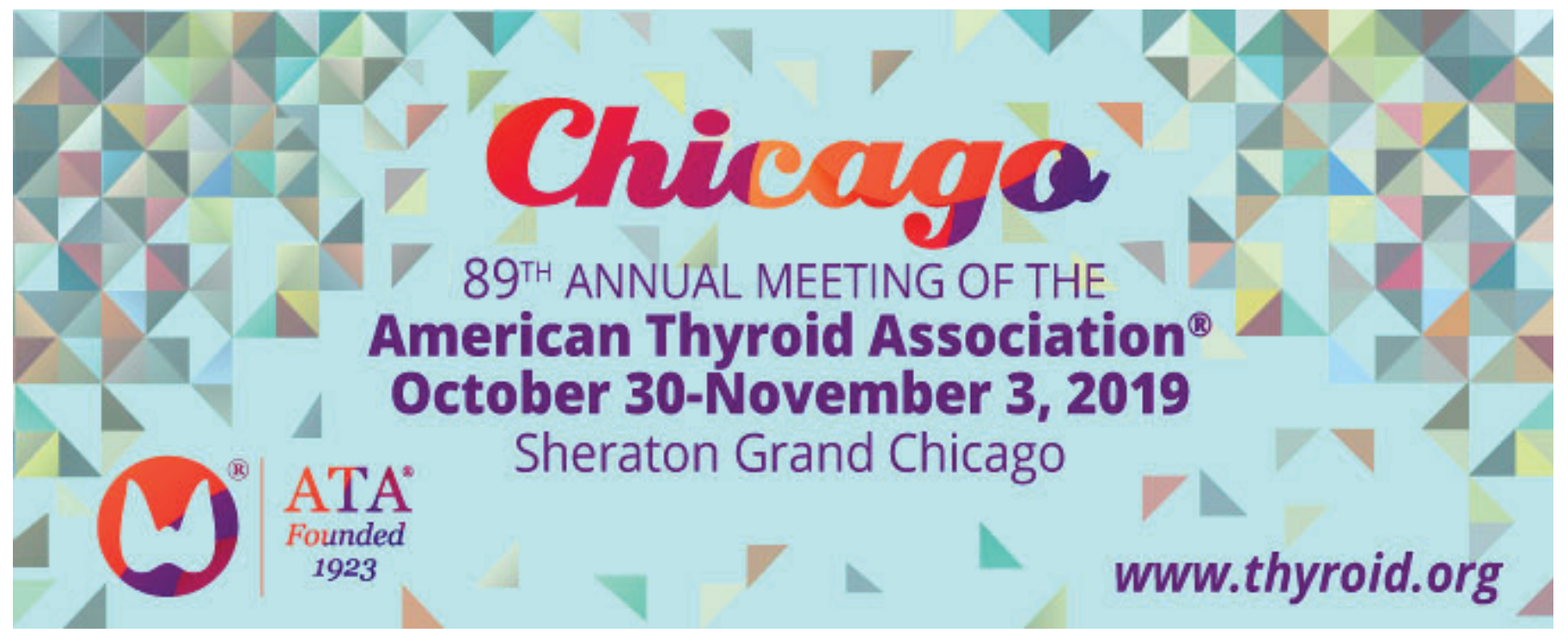

